\title{
Glutathione as a Prognostic Biomarker and a Potential Therapeutic Target for Ovarian Cancer
}

\section{Motoki Takenaka1 ${ }^{*}$, Tatsuro Furui ${ }^{1}$, Noriko Suzuki ${ }^{1}$, Tiger Koike1, Hitomi Aoki², Ken-Ichirou Morishige ${ }^{1}$}

${ }^{1}$ Department of Obstetrics and Gynecology, Gifu University School of Medicine, Gifu, Japan

${ }^{2}$ Department of Tissue and Organ Development, Gifu University Graduate School of Medicine, Gifu, Japan

Email: *bridge@gifu-u.ac.jp

How to cite this paper: Takenaka, M., Furui, T., Suzuki, N., Koike, T., Aoki, H. and Morishige, K.-I. (2022) Glutathione as a Prognostic Biomarker and a Potential Therapeutic Target for Ovarian Cancer. Open Journal of Obstetrics and Gynecology, 12, 56-66.

https://doi.org/10.4236/ojog.2022.121006

Received: December 22, 2021

Accepted: January 21, 2022

Published: January 24, 2022

Copyright $\odot 2022$ by author(s) and Scientific Research Publishing Inc. This work is licensed under the Creative Commons Attribution International License (CC BY 4.0).

http://creativecommons.org/licenses/by/4.0/

\begin{abstract}
Aim: Glutathione (GSH) is an antioxidant, protecting cell against toxicity of reactive oxygen species (ROS). Data showed that GSH might play roles in malignancy including ovarian cancer (OC), and, thus, we attempted to determine the clinical significance of GSH and effects of erastin (an inhibitor of GSH synthesis) in OC. Methods: OC tissues were taken from 41 OC patients, and cancer-tissue GSH level was measured with GSH Assay Kit. Survival curves were carried out by the Kaplan-Meier method and evaluated using the log-rank test. Multivariable Cox proportional hazard risk regression model was performed to screen the independent factor affecting the prognosis of OC patients. In vitro effect of erastin was studied using OC cell lines. Cell viability, GSH levels and whole (cytosolic and lipid) ROS production were assessed. Results: Patients with high OC-tissue-GSH levels had an apparently lower progression free survival (PFS) and overall survival (OS) compared with those with low GSH levels. The GSH levels were independent factors for predicting the PFS and OS. The basal ROS level was inversely proportional to GSH levels in OC cell lines. The basal GSH levels were important for estimating the sensitivity to erastin. Reduction of intracellular GSH levels increased whole ROS, which caused cell deaths. Conclusions: Data suggested that the GSH levels could be a candidate of prognostic biomarkers and that erastin might be worth studying as a new therapeutic drug in OC.
\end{abstract}

\section{Keywords}

Glutathione, Reactive Oxygen Species, Ovarian Cancer, Erastin 


\section{Introduction}

The number of patients with ovarian cancer (OC) is increasing and deaths due to this disease are also increasing in Japan [1]. Platinum compound is one of the most potent chemotherapy drugs widely used for OC [2] [3]. Although OC is generally sensitive to chemotherapy, most patients ultimately recur and develop resistance to chemotherapy [4] [5]. Survival rates are estimated based on previous outcomes [6], but it is not easy to predict what will happen in OC patients.

Glutathione (GSH), an antioxidant, is used to mitigate the damage of reactive oxygen species (ROS) [7]. They have roles in cell cycle progression and cell death pathway. Erastin is a classical inhibitor that can lead to the depletion of GSH [8].

In this study, we focused on a potential of GSH as a new prognostic biomarker and erastin as a new strategy in anti-tumor therapies for ovarian cancer.

\section{Materials \& Methods}

\subsection{Patients}

A total of 41 OC tissues were obtained from patients who underwent operation between 2009 and 2018 at Gifu university hospital. The OC specimens were subjected to histological examination by 2 expert pathologists for confirmation of World Health Organization (WHO) classification of the tumor, and staging according to the tumor-node-metastasis (TNM) system. The ethics committee of the Gifu University Graduate School of Medicine approved the experiments. Written informed consent was obtained from all patients.

\subsection{GSH Analysis in Human Ovarian Cancer Tissues}

We performed GSH analysis using the specimen prepared by freezing the tissue separated. We took central and deep into the OC tissues as samples from the patient during operation. The tissues were lysed in 5\% 5-sulfosalicylic acid dihydrate (Wako Pure Chemical Industries). The lysate was centrifuged $\left(1000{ }^{\star} \mathrm{g}\right)$ and the supernatant was collected. The supernatant was used to determine the amount of GSH in the sample. We used the GSH and GSSG Assay Kit (product No. S0053, Beyotime) and followed the product instructions to determine GSH levels. Briefly, GSH assay buffer, GSH reductase, 5,5'-dithio-bis 2-nitrobenzoic acid solution and supernatant sample were mixed together and incubated at $25^{\circ} \mathrm{C}$ for 5 minutes, then NADPH was added into this system to trigger the reaction. The increase in the absorbance of 5-thio-2-nitrobenzoic acid was measured at $412 \mathrm{~nm}$, and the GSH levels were calculated following the product instructions.

\subsection{Cell Culture}

OC cell lines, TOV-21G, KOC-7C (clear cell carcinoma), CaOV3 and SKOV3ip1 (serous carcinoma) were used. KOC-7C cells were provided by the Gynecological and Obstetrics Department, Kurume University and others were provided by the Gynecological and Obstetrics Department, Osaka University [9]. These cells 
were cultured in Dulbecco's modified medium (Wako) containing $10 \%$ fetal bovine serum (FBS) and $1 \%$ penicillin/streptomycin (Wako) under $5 \% \mathrm{CO}_{2}$ at $37^{\circ} \mathrm{C}$.

\subsection{Drug Treatments}

Treatments of OC cell lines with $2.5-20 \mu \mathrm{M}$ erastin, an inhibitor of GSH synthesis, or 0.5\% DMSO as control were performed in Dulbecco's modified medium containing $10 \% \mathrm{FBS}$ and $1 \%$ penicillin/streptomycin.

\subsection{Cell Viability Analysis}

Cell viability was evaluated using a Premix WST-1 Cell Proliferation Assay System (TaKaRa Bio Incorporated) according to the manufacturer's instructions. Briefly, cells $\left(1 \times 10^{4}\right.$ cells/well $)$ were seeded in a 96-well plate and treated with different drugs at various concentrations for the indicated times. After addition of $10 \mu \mathrm{l}$ Premix WST-1 solution to each well, cells were incubated at $37^{\circ} \mathrm{C}$ for another 1 hour and the absorbance was determined at $440 \mathrm{~nm}$ using a microplate reader.

\subsection{Intracellular GSH Analysis}

Cells were plated in 6-well plates at a density of $3.0 \times 10^{5}$ cells/well and cultured overnight. Cells were received different treatment for 4 hours followed by harvesting to determine cell number. Nearly $6 \times 10^{4}$ live cells from each sample were transferred to new tubes, washed in phosphate buffered saline (PBS) and centrifuged at $1200 \mathrm{rpm}$ at $4^{\circ} \mathrm{C}$ for 5 minutes twice. The cell pellet was resuspended in $80 \mu \mathrm{l}$ protein removal solution, thoroughly incorporated, and placed in $-70^{\circ} \mathrm{C}$ and $37^{\circ} \mathrm{C}$ sequentially for fast freezing and thawing, then placed in $4^{\circ} \mathrm{C}$ for 5 minutes and centrifuged at $10,000{ }^{*} \mathrm{~g}$ for 10 minutes. The supernatant was used to determine the amount of GSH in the sample. We used the GSH and GSSG Assay Kit and followed the product instructions to determine GSH levels.

\subsection{Analysis of ROS Production}

Cells were plated in $10 \mathrm{~cm}$ dishes at a density of $1.0 \times 10^{5}$ cells/well and cultured overnight. After treated with test compounds for 15 hours, harvested in $5 \mathrm{ml}$ Dulbecco's modified medium containing Deep Red Reagent (5 $\mu \mathrm{M}$ ) (Molecular Probes, Invitrogen) and incubated for $30 \mathrm{~min}$ at $37^{\circ} \mathrm{C}$ in a tissue culture incubator. After trypsinized, cells were resuspended in 3\% FBS in PBS and strained through a $40 \mathrm{mM}$ cell strainer (BD Falcon). Cells were analyzed using a flow cytometer (FACS Aria, BD Biosciences) equipped with $488 \mathrm{~nm}$ laser for excitation. Data were collected from the Deep Red Reagent channel (MitoSOX). A minimum of $1.0 \times 10^{4}$ cells were analyzed per condition.

\subsection{Statistical Analysis}

Data are expressed as means \pm SD of 3 independent experiments and were evaluated using an ANOVA LSD test. Student $t$ test was conducted for intergroup 
comparison. Pearson correlation test was used for correlation analysis. Survival curves were generated with Kaplan-Meier plots. The results are presented with $P$ values from a log-rank test. Multivariable Cox proportional hazard risk regression model was performed to screen the independent factor affected the prognosis of OC patients. All tests were 2 -sided, and $P$ values $<0.05$ were considered statistically significant.

\section{Results}

\subsection{Patient Characteristics}

The GSH levels in 41 OC tissues were measured. The GSH levels were considered as either low $(n=34)$ or high $(n=7)$ according to the cut-off value, which was defined as the median of the cohort. The GSH levels were $73.1 \pm 74.1$ and $669.1 \pm 339.5 \mu \mathrm{mol} / \mathrm{l}$ in each low and high group (Table 1 ). There was no significant difference in all parameters (e.g., age, type of pathology, stage, type of surgery, neoadjuvant and adjuvant chemotherapy).

Table 1. Characteristics of ovarian cancer patients who received GSH levels assay.

\begin{tabular}{|c|c|c|c|}
\hline & \multicolumn{2}{|c|}{ GSH levels } & \multirow[b]{2}{*}{$P$-value } \\
\hline & low & high & \\
\hline Cases (n) & 34 & 7 & \\
\hline GSH levels (average \pm SD) & $73.1 \pm 74.1$ & $669.1 \pm 339.5$ & $<0.01$ \\
\hline age (average $\pm \mathrm{SD}$ ) & $60.0 \pm 11.0$ & $60.0 \pm 16.5$ & 0.55 \\
\hline type of pathology & & & 0.26 \\
\hline HGSC & 13 & 5 & \\
\hline LGSC & 1 & 0 & \\
\hline $\mathrm{CCC}$ & 17 & 1 & \\
\hline $\mathrm{EC}$ & 1 & 1 & \\
\hline $\mathrm{MC}$ & 2 & 0 & \\
\hline Stage & & & 0.73 \\
\hline $\mathrm{I}+\mathrm{II}$ & 17 & 3 & \\
\hline $\mathrm{III}+\mathrm{IV}$ & 17 & 4 & \\
\hline Type of surgery & & & 0.85 \\
\hline complete and optimal & 30 & 6 & \\
\hline Others & 4 & 1 & \\
\hline NAC & & & 0.82 \\
\hline+ & 13 & 3 & \\
\hline- & 21 & 4 & \\
\hline adjuvant chemotherapy & & & 0.41 \\
\hline+ & 31 & 7 & \\
\hline- & 3 & 0 & \\
\hline
\end{tabular}

HGSC = high grade serous carcinoma, LGSC = low grade serous carcinoma, CCC $=$ clear cell carcinoma, $\mathrm{EC}=$ endometrioid carcinoma, $\mathrm{MC}=$ mucinous carcinoma, $\mathrm{NAC}=$ neoadjuvant chemotherapy. 


\subsection{High GSH Levels Predict Poor Prognosis in OC}

To investigate the clinical significance of GSH, we assessed the association GSH levels and prognosis in OC. The Kaplan-Meier analysis was performed to determine the prognostic value of GSH in OC patients. We performed a log-rank test which compared patients with higher GSH levels with those with lower levels. Patients with higher GSH levels had significantly shorter progression free survival (PFS) than those with a lower GSH levels $(P<0.01)$ (Figure 1$)$. It also showed that the overall survival (OS) was shorter in OC patients with higher GSH levels $(P<0.001)$ (Figure 2$)$.

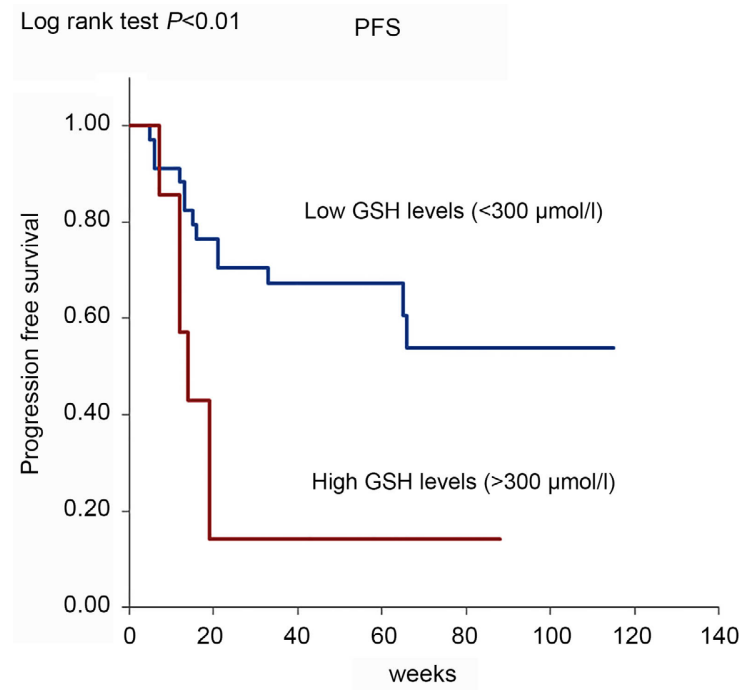

Figure 1. The Kaplan-Meier progression free survival determined by GSH levels of OC patients. According to the GSH levels, Kaplan-Meier progression free survival of OC patients showed that high GSH levels were significantly correlated with poor prognosis $(P<0.01)$.

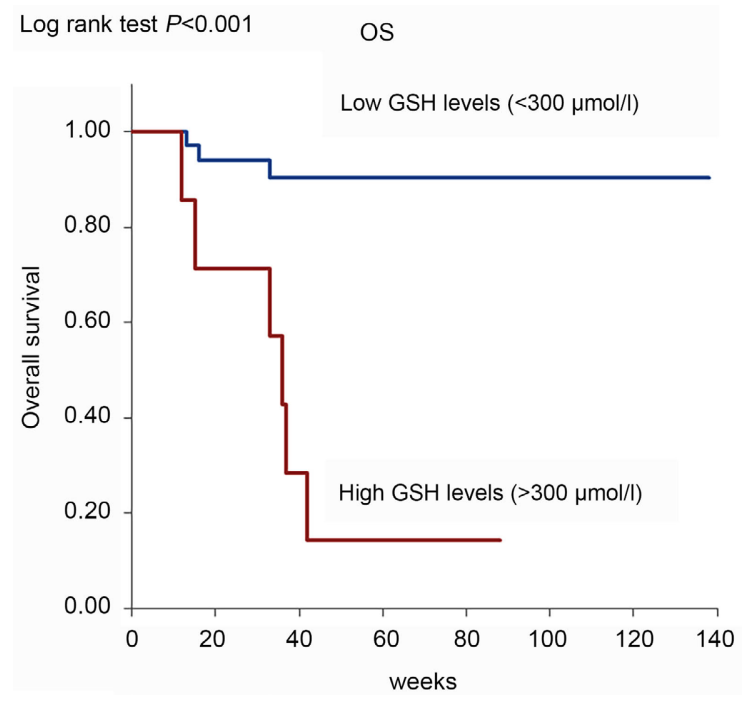

Figure 2. The Kaplan-Meier overall survival determined by GSH levels of OC patients. According to the GSH levels, Kaplan-Meier overall survival of OC patients showed that high GSH levels were significantly correlated with poor prognosis $(P<0.001)$. 


\subsection{GSH Levels as Independent Factors for Predicting the Prognosis of OC}

We performed the multivariate Cox regression analysis to show that GSH levels were independent factors predicting the prognosis. GSH levels were independent factors for both PFS (HR = 5.330, 95\% CI: $1.334-21.291 ; P=0.018)$ and OS (HR $=7.174,95 \%$ CI: $1.572-32.738 ; P=0.011$ ) (Table 2 and Table 3 ).

\subsection{The Sensitivity to an Inhibitor of GSH Synthesis, Erastin, in OC Cell Lines}

Next, we demonstrated in vitro investigation using OC cell lines focused on GSH. Erastin is identified as an inhibitor of GSH synthesis. To determine whether erastin induces growth inhibition in OC cells, TOV-21G, KOC-7C, CaOV3 and SKOV3ip1 cells were treated with erastin for 24 hours and cell viability was assayed using Premix WST-1 assay (Figure 3). Comparatively, TOV-21G and SKOV3ip1 cells were more sensitive to erastin than CaOV3 and KOC-7C cells.

Table 2. Multivariate analysis for progression free survival by Cox regression model.

\begin{tabular}{cccccc}
\hline Variable & Hazard ratio & \multicolumn{3}{c}{$95 \%$ CI } & $P$-value \\
\hline GSH levels & 7.174 & 1.572 & - & 32.738 & 0.011 \\
age & 0.976 & 0.926 & - & 1.028 & 0.354 \\
type of pathology & 3.834 & 0.972 & - & 15.126 & 0.055 \\
stage & 4.440 & 0.541 & - & 36.439 & 0.165 \\
type of surgery & 0.170 & 0.030 & - & 0.974 & 0.047 \\
NAC & 3.191 & 0.660 & - & 15.437 & 0.149 \\
adjuvant chemotherapy & 1.049 & 0.028 & - & 39.331 & 0.979
\end{tabular}

$\mathrm{CI}=$ confidence interval, $\mathrm{NAC}=$ neoadjuvant chemotherapy.

Table 3. Multivariate analysis for overall survival by Cox regression model.

\begin{tabular}{cccccc}
\hline Variable & Hazard ratio & \multicolumn{3}{c}{$95 \%$ CI } & $P$-value \\
\hline GSH levels & 5.330 & 1.334 & - & 21.291 & 0.018 \\
age & 0.992 & 0.953 & - & 1.032 & 0.683 \\
type of pathology & 3.244 & 0.839 & - & 12.539 & 0.088 \\
stage & 2.526 & 0.374 & - & 17.061 & 0.342 \\
type of surgery & 0.233 & 0.045 & - & 1.198 & 0.081 \\
NAC & 2.833 & 0.675 & - & 11.882 & 0.155 \\
adjuvant chemotherapy & 1.632 & 0.066 & - & 40.081 & 0.764 \\
\hline
\end{tabular}

$\mathrm{CI}=$ confidence interval, $\mathrm{NAC}=$ neoadjuvant chemotherapy. 


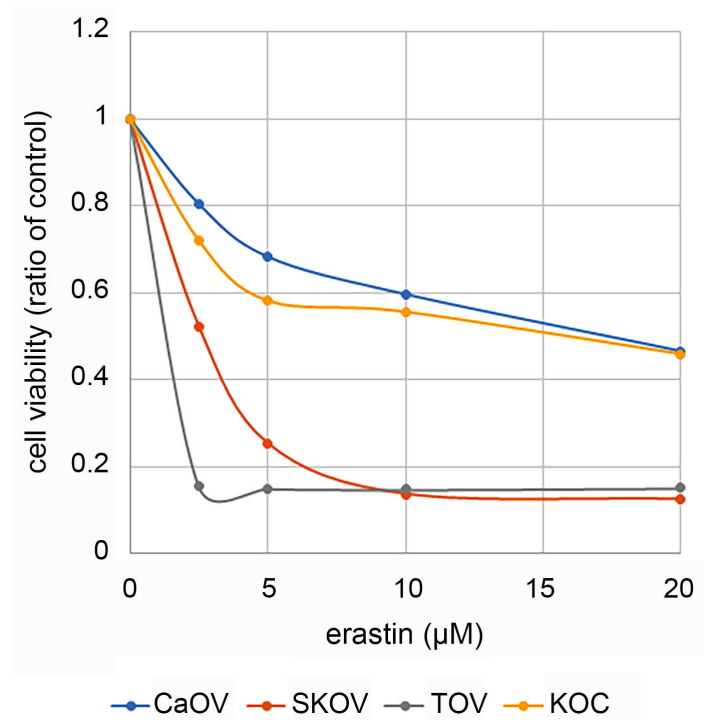

Figure 3. The sensitivity to erastin in OC cell lines. OC cell lines (CaOV, SKOV, TOV-21G and KOC-7C) were treated with erastin for 24 hours and cell viability was assayed using Premix WST-1 assay.

\subsection{The Accumulation of ROS Induced by GSH Depletion}

We compared erastin sensitive OC cell line, TOV-21G, with less sensitive cell line, KOC-7C, focused on the basal GSH and ROS level. Although the GSH levels were reduced by erastin both in TOV-21G and KOC-7C cell, the basal GSH levels in KOC-7C cells were higher than that in TOV-21G cells under control condition (Figure 4). We observed that treatment with erastin resulted in increase of ROS in both cell lines but the basal ROS level in KOC-7C cell was lower than that in TOV-21G cell (Figure 5).

Erastin decreased intracellular GSH levels and increased ROS resulting in cell death in OC cells. The sensitivity to erastin was depended on intracellular basal GSH levels.

\section{Discussions}

Oxidative stress has long been implicated in cancer development and progression [10], suggesting that antioxidant treatment may provide protection from cancer [11]. Among the enzymatic systems involved in the maintenance of the intracellular redox balance, a main role is played by GSH that participates not only in antioxidant defense systems but also in many metabolic processes [12] [13]. GSH is a tripeptide formed by glutamic acid, cysteine, and glycine. In many normal and malignant cells, increased GSH levels are associated with a proliferative response and essential for cell cycle progression [14] [15]. On the other hand, GSH depletion regulates the activation of cell death pathways [16].

Thus, several studies about biochemical function of GSH were published. However, their potential utility in clinical practice as prognostic biomarkers is unknown. To investigate the clinical features of the GSH status, we assessed the associations between GSH levels and prognosis of OC patients. The GSH levels 


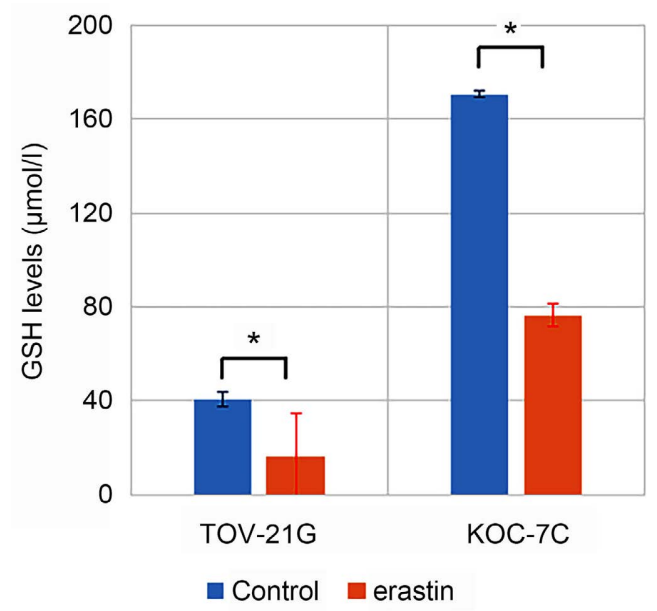

Figure 4. GSH levels influenced by erastin. TOV-21G and KOC-7C cells were treated with erastin $(10 \mu \mathrm{M})$ for 4 hours and GSH levels were assayed using GSSG/GSH Quantification Kit. ${ }^{*} P<0.05$, Student's $t$ test.

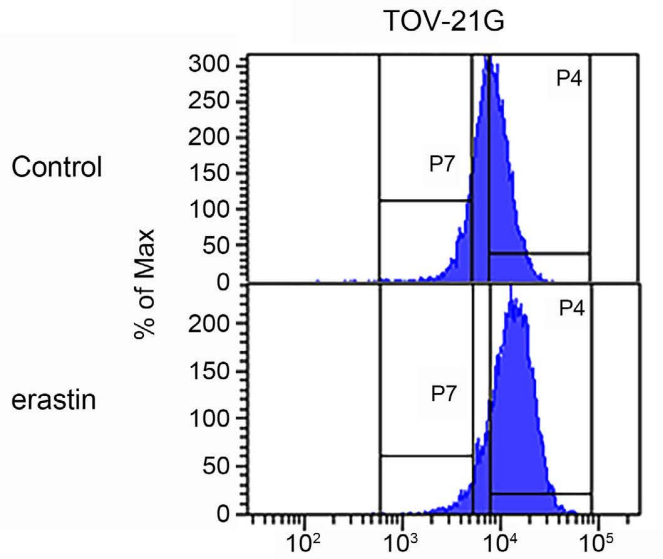

Deep Red Reagent fluorescence fluo

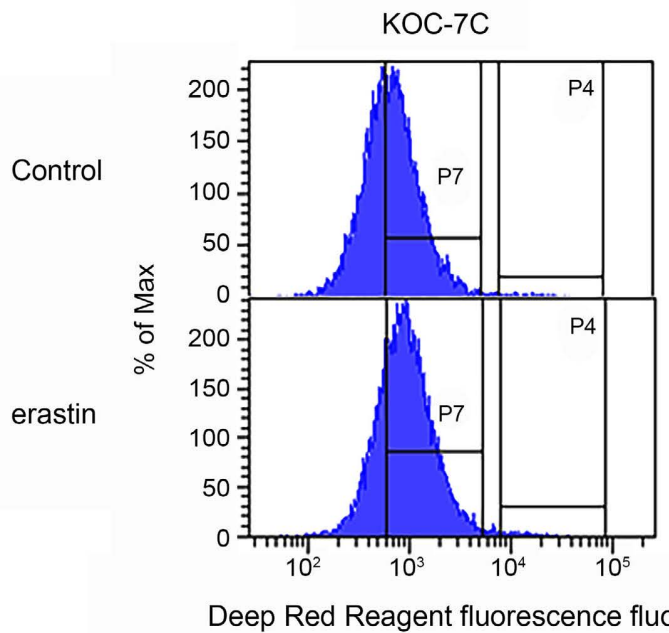

Figure 5. ROS level influenced by erastin. TOV-21G and KOC-7C cells were treated with erastin $(10 \mu \mathrm{M})$ for 15 hours. Following, whole (cytosolic and lipid) ROS production were assessed by flow cytometry using Deep Red Reagent. 
in OC tissues of 41 patients were measured. Interestingly, OC patients with high GSH levels had an evidently lower PFS and OS compared with those with low GSH levels. Notably, GSH levels were independent factors for predicting the PFS and OS of OC patients. We investigated that elevated GSH levels lead antioxidant defense systems resulting in defense to evade cancer cell death and develop drug resistance. Our data indicated that GSH might be a potent biomarker for predicting the prognosis of $\mathrm{OC}$ patients.

Next, we demonstrated in vitro investigation to assess the oxidant system associated with GSH levels in OC. Recently, Qi Cheng et al. [17] reported that erastin works synergistically with cisplatin to inhibit OC cell growth. Erastin induced the depletion of GSH resulting in increase of lipid ROS [18] [19]. In detail, erastin inhibits system Xc resulting in cysteine starvation and ferroptotic cell death [20] [21].

Our data showed that the basal GSH levels were lower in erastin sensitive OC cell lines than less sensitive cell lines and the basal ROS level was inversely proportional to GSH levels in both cell lines. Although the depletion of GSH induced by erastin leads to ROS in both cell lines, cell death could not be lead in less sensitive OC cell lines because the basal ROS level was too low. We showed that the basal GSH levels are important for estimating the sensitivity to erastin.

\section{Conclusion}

Our study demonstrated that high GSH levels were significantly associated with a poor prognosis independently of other factors in OC. Erastin leads OC cell death depend on intracellular GSH levels. GSH could be a prognostic biomarker and erastin might be worth studying as a new drug of OC treatment.

\section{Acknowledgements}

We gratefully acknowledge the work of past and present members of our laboratory.

\section{Conflicts of Interest}

The authors declare no conflicts of interest regarding the publication of this paper.

\section{References}

[1] National Cancer Center (2021) Cancer Statistics in Japan. https://ganjoho.jp/reg_stat/statistics/data/dl/en.html

[2] Ozols, R.F., Bundy, B.N., Greer, B.E., Fowler, J.M., Clarke-Pearson, D., Burger, A.R., Mannel, R.S., DeGeest, K., Hartenbach, E.M., Baergen, R. and Gynecologic Oncology Group (2003) Phase III Trial of Carboplatin and Paclitaxel Compared with Cisplatin and Paclitaxel in Patients with Optimally Resected Stage III Ovarian Cancer: A Gynecologic Oncology Group Study. Journal of Clinical Oncology, 21, 3194-3200. https://doi.org/10.1200/JCO.2003.02.153

[3] Bois, A., Lück, H.J., Meier, W., Adams, H.P., Mobus, V., Costa, S., Bauknecht, T., 
Richter, B., Warm, M., Schröder, W., Olbricht, S., Nitz, U., Jackisch, C., Emons, G., Wagner, U., Kuhn, W., Pfisterer, J. and Arbeitsgemeinschaft Gynäkologische Onkologie Ovarian Cancer Study Group (2003) A Randomized Clinical Trial of Cisplatin/Paclitaxel versus Carboplatin/Paclitaxel as First-Line Treatment of Ovarian Cancer. Journal of the National Cancer Institute, 95, 1320-1329.

https://doi.org/10.1093/jnci/djg036

[4] Heintz, A.P., Odicino, F., Maisonneuve, P., Beller, U., Benedet, J.L., Creasman, W.T., Ngan, H.Y.S., Pecorelli, S. and Belleret, U. (2006) Carcinoma of the Ovary. FIGO 26th Annual Report on the Results of Treatment in Gynecological Cancer. International Journal of Gynecology \& Obstetrics, 95, 161-192. https://doi.org/10.1016/S0020-7292(06)60033-7

[5] Markman, M., Markman, J., Webster, K., Zanotti, K., Kulp, B., Peterson, G. and Belinson, J. (2004) Duration of Response to Second-Line, Platinum-Based Chemotherapy for Ovarian Cancer: Implications for Patient Management and Clinical Trial Design. Journal of Clinical Oncology, 22, 3120-3125.

https://doi.org/10.1200/JCO.2004.05.195

[6] Roett, M.A. and Evans, P. (2009) Ovarian Cancer: An Overview. American Academy of Family Physicians, 80, 609-616.

[7] Dixon, S.J., Lemberg, K.M., Lamprecht, M.R., Skouta, R., Zaitsev, E.M., Gleason, C.E., Patel, D.N., Bauer, A.J., Cantley, A.M., Yang, W.S., Morrison, B. and Stockwell, B.R. (2012) Ferroptosis: An Iron-Dependent form of Nonapoptotic Cell Death. Cell, 149, 1060-1672. https://doi.org/10.1016/j.cell.2012.03.042

[8] Sun, Y., Deng, R. and Zhang, C. (2020) Erastin Induces Apoptotic and Ferroptotic Cell Death by Inducing ROS Accumulation by Causing Mitochondrial Dysfunction in Gastric Cancer Cell HGC-27. Journal of Molecular Medicine, 22, 2826-2832. https://doi.org/10.3892/mmr.2020.11376

[9] Tomioka, Y. (1998) Establishment and Characterization of Three Human Ovarian Clear Cell Carcinoma Cell Lines (KOC-4c, KOC-5c, KOC-7c). The Journal of the Kurume Medical Association, 61, 323-333.

[10] Hussain, S.P., Hofseth, L.J. and Harris, C.C. (2003) Radical Causes of Cancer. Nature Reviews Cancer, 3, 276-285. https://doi.org/10.1038/nrc1046

[11] Cabello, C.M., Bair, W.B. and Wondrak, G.T. (2007) Experimental Therapeutics: Targeting the Redox Achilles Heel of Cancer. Current Opinion in Pharmacology, 8, 1022-1037.

[12] Meister, A. (1995) Glutathione Metabolism. Methods in Enzymology, 251, 3-7. https://doi.org/10.1016/0076-6879(95)51106-7

[13] Sies, H. (1999) Glutathione and Its Role in Cellular Functions. Free Radical Biology \& Medicine, 27, 916-921. https://doi.org/10.1016/S0891-5849(99)00177-X

[14] Messina, J.P. and Lawrence, D.A. (1989) Cell Cycle Progression of GlutathioneDepleted Human Peripheral Blood Mononuclear Cells Is Inhibited at S Phase. The Journal of Immunology, 143, 1974-1981.

[15] Lu, S.C. and Ge, J.L. (1992) Loss of Suppression of GSH Synthesis at Low Cell Density in Primary Cultures of Rat Hepatocytes. American Physiological Society Journal, 263, 1181-1189. https://doi.org/10.1152/ajpcell.1992.263.6.C1181

[16] Franco, R. and John, A. (2012) Glutathione Efflux and Cell Death. Antioxidants \& Redox Signaling, 17, 1694-1713. https://doi.org/10.1089/ars.2012.4553

[17] Cheng, Q., Bao, L., Li, M., Chang, K. and Yi, X. (2021) Erastin Synergizes with Cisplatin via Ferroptosis to Inhibit Ovarian Cancer Growth in Vitro and in Vivo. Journal of Obstetrics and Gynaecology Research, 47, 2481-2491. 
https://doi.org/10.1111/jog.14779

[18] Mehta, A., Prabhakar, M., Kumar, M., Deshmukh, R. and Sharma, P. (2013) Excitotoxicity: Bridge to Various Triggers in Neurodegenerative Disorders. European Journal of Pharmacology, 698, 6-18. https://doi.org/10.1016/j.ejphar.2012.10.032

[19] Bridges, R., Natale, N. and Patel, S. (2012) System Xc-cystine/Glutamate Antiporter: An Update on Molecular Pharmacology and Roles within the CNS. British Journal of Pharmacology, 165, 20-34. https://doi.org/10.1111/j.1476-5381.2011.01480.x

[20] Bannai, S. and Kitamura, E. (1980) Transport Interaction of L-cystine and L-glutamate in Human Diploid Fibroblasts in Culture. Journal of Biological Chemistry, 255, 2372-2376. https://doi.org/10.1016/S0021-9258(19)85901-X

[21] Tan, S., Schubert, D. and Maher, P. (2001) Oxytosis: A Novel form of Programmed Cell Death. Current Topics in Medicinal Chemistry, 1, 497-506.

https://doi.org/10.2174/1568026013394741 